University of New Orleans

ScholarWorks@UNO

$10-2004$

\title{
Achromatic angle-insensitive infrared quarter-wave retarder based on total internal reflection at the $\mathrm{Si}-\mathrm{SiO} 2$ interface
}

\author{
R. M.A. Azzam \\ University of New Orleans, razzam@uno.edu \\ Cristina L. Spinu \\ cspinu@uno.edu
}

Follow this and additional works at: https://scholarworks.uno.edu/ee_facpubs

Part of the Electrical and Electronics Commons, and the Optics Commons

\section{Recommended Citation}

R. M. A. Azzam and Cristina L. Spinu, "Achromatic angle-insensitive infrared quarter-wave retarder based on total internal reflection at the Si-SiO2 interface," J. Opt. Soc. Am. A 21, 2019-2022 (2004)

This Article is brought to you for free and open access by the Department of Electrical Engineering at ScholarWorks@UNO. It has been accepted for inclusion in Electrical Engineering Faculty Publications by an authorized administrator of ScholarWorks@UNO. For more information, please contact scholarworks@uno.edu. 


\title{
Achromatic angle-insensitive infrared quarter-wave retarder based on total internal reflection at the $\mathrm{Si}-\mathrm{SiO}_{2}$ interface
}

\author{
R. M. A. Azzam and Cristina L. Spinu \\ Department of Electrical Engineering, University of New Orleans, New Orleans, Louisiana 70148
}

Received March 31, 2004; revised manuscript received May 19, 2004; accepted May 21, 2004

An achromatic infrared $(\lambda=1.2-4 \mu \mathrm{m})$, Si-prism quarter-wave retarder (QWR) is described that uses total internal reflection at a buried $\mathrm{Si}-\mathrm{SiO}_{2}$ interface at an angle of incidence $\phi$ near $33^{\circ}$, where $\partial \Delta / \partial \phi=0$. The retardance $\Delta$ deviates from $90^{\circ}$ by $< \pm 2^{\circ}$ within a field of view of $\pm 10^{\circ}$ (in air) over the entire bandwidth. Because the $\mathrm{SiO}_{2}$ layer at the base of the prism is optically thick, this QWR is unaffected by environmental contamination. (C) 2004 Optical Society of America

OCIS codes: $230.0230,230.5440,240.0240,260.2130,260.5430$.

\section{INTRODUCTION}

Quarter-wave retarders (QWRs) are widely used for polarization control and analysis in many applications, ${ }^{1-3}$ and their development is of continuing interest. ${ }^{4-8}$ The desired differential phase shift $\Delta=\pi / 2$ between the two orthogonal linear eigenpolarizations of the device is often achieved in light transmission through a section of a transparent, birefringent medium. The birefringence may be natural (e.g., as in a mica sheet, quartz plate, or an oriented liquid-crystal layer), induced in an otherwise isotropic medium by an applied stress or electric field, or may be artificial, e.g., by creating a subwavelengthstructured grating ${ }^{9,10}$ or photonic crystal. ${ }^{5}$ However, it is difficult to make birefringent QWRs achromatic. ${ }^{4}$ By using multilayer interference coatings at oblique incidence, ${ }^{11-14}$ it is possible to design QWRs that are achromatic within a small bandwidth.

By far the most achromatic QWRs are those based on total internal reflection (TIR). ${ }^{15,16}$ Here we present a new, broadband (wavelength $\lambda=1.2-4 \mu \mathrm{m}$ ), Si-prism QWR (Fig. 1) that uses a single TIR at a buried $\mathrm{Si}-\mathrm{SiO}_{2}$ interface. By operating near the point at which $\partial \Delta / \partial \phi$ $=0$, where $\phi$ is the angle of incidence, the retardance $\Delta$ deviates from quarter-wave by $< \pm 2^{\circ}$ within a field of view (in air) of $\pm 10^{\circ}$. Because TIR occurs at an embedded interface (the oxide layer is optically thick), the device is completely insensitive to environmental contamination. Given that the $\mathrm{Si}-\mathrm{SiO}_{2}$ system is perhaps the most well characterized material system, this device may be suited for use as a standard reference QWR in the infrared. (However, this requires that birefringence due to any residual stresses in the Si be minimized.) In this regard we note that Si optics has been gaining considerable attention recently. ${ }^{17,18}$

\section{ANGLE-INSENSITIVE QUARTER-WAVE RETARDER THAT USES TOTAL INTERNAL REFLECTION AT A DIELECTRIC-AIR INTERFACE}

For TIR at angles of incidence $\phi$ above the critical angle $\phi_{c}=\arcsin \left(n_{1} / n_{0}\right)$, the differential reflection phase shift $\Delta=\delta_{p}-\delta_{s}$ of $p$ - and $s$-polarized light is given by ${ }^{3,19}$

$$
\tan (\Delta / 2)=\left(n^{2} \sin ^{2} \phi-1\right)^{1 / 2} /(n \sin \phi \tan \phi),
$$

where

$$
n=n_{0} / n_{1}
$$

is the high-to-low ratio of refractive indices of the media of incidence and (evanescent) refraction, respectively ( $n$ $>1$ ). To satisfy the following two conditions, ${ }^{15,20}$

$$
\Delta=90^{\circ}, \quad \partial \Delta / \partial \phi=0,
$$

we must select

$$
\begin{aligned}
& n=\sqrt{2}+1=2.414214, \\
& \phi=\phi_{90}=\arccos \left(2^{-1 / 4}\right)=32.7651^{\circ} .
\end{aligned}
$$

Figure 2 is a plot of $\Delta$ versus $\phi$ using Eq. (1) for $n=\sqrt{2}$ +1 , which confirms that Eqs. (3) are satisfied at the angle $\phi_{90}$.

To our knowledge, there has been only one previous suggestion $^{15}$ of using TIR at a diamond-air interface to satisfy Eqs. (3) and (4). Indeed if a published dispersion relation for diamond ${ }^{21}$ (C) is used, we find that $n=\sqrt{2}$ +1 at wavelength $\lambda=614.35 \mathrm{~nm}$ in the visible. However, optical-quality bulk diamond is expensive, which makes this proposition impractical. One practical infra- 


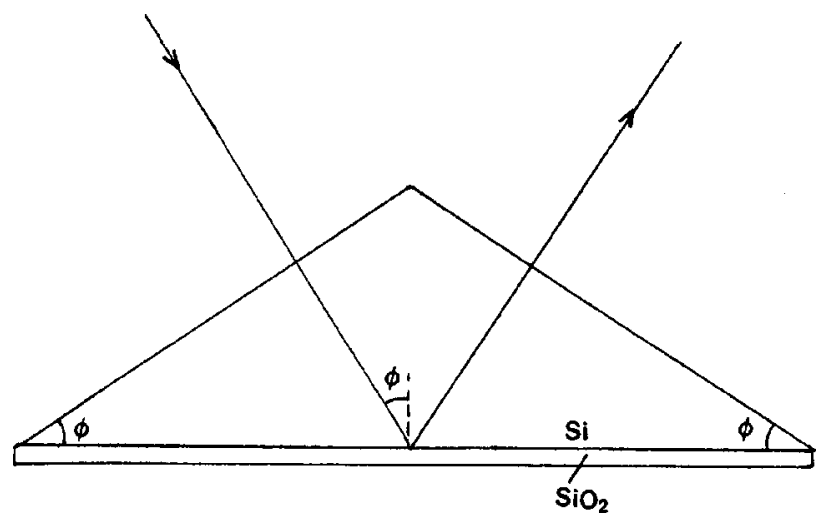

Fig. 1. QWR that uses total internal reflection at the base of a Si prism and that is coated with an optically thick $\mathrm{SiO}_{2}$ film. The entrance and exit faces are antireflection coated. The optimum operating angle is $\phi=32.765^{\circ}$.

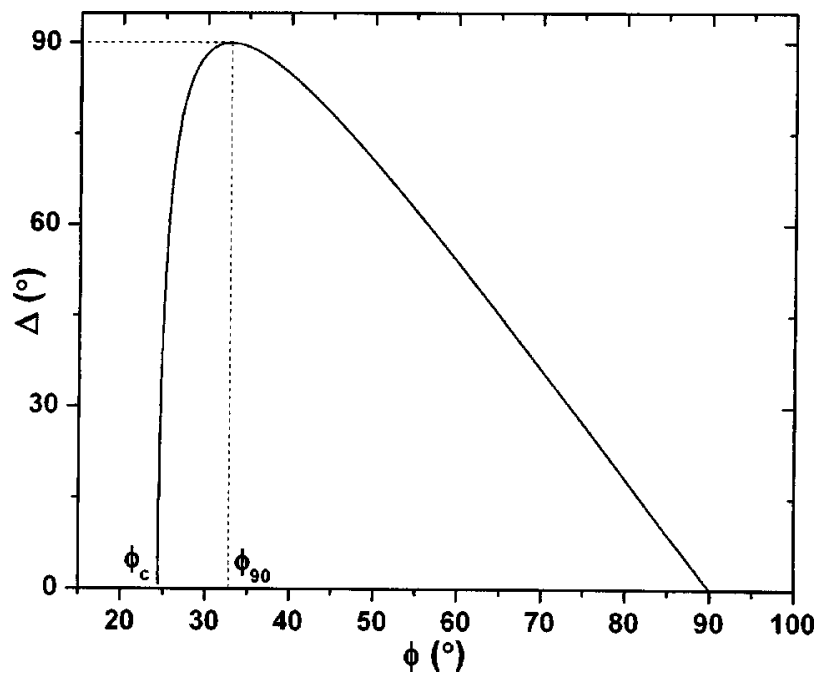

Fig. 2. Plot of $\Delta$ versus $\phi$ [by use of Eq. (1)] for $n=\sqrt{2}+1$ confirms the conditions represented by Eqs. (3) and (4).

red design employs TIR at a $\mathrm{ZnSe}$-air interface. From the dispersion formula for $\mathrm{ZnSe},{ }^{21}$ we find that $n=\sqrt{2}$ +1 at $\lambda=8.6156 \mu \mathrm{m}$.

\section{ACHROMATIC QUARTER-WAVE RETARDER THAT USES TOTAL INTERNAL REFLECTION AT THE Si-SiO INTERFACE}

The achromatic design that we propose in this paper uses TIR at the buried $\mathrm{Si}-\mathrm{SiO}_{2}$ interface. The refractive indices of low-doped $\mathrm{Si}$ and $\mathrm{SiO}_{2}$ (fused silica) are given by ${ }^{21}$

$$
\begin{aligned}
n^{2}(\mathrm{Si})= & 1+\frac{10.6684293 \lambda^{2}}{\lambda^{2}-(0.301516485)^{2}} \\
& +\frac{0.003043475 \lambda^{2}}{\lambda^{2}-(1.13475115)^{2}}+\frac{1.54133408 \lambda^{2}}{\lambda^{2}-(1104.0)^{2}}
\end{aligned}
$$

$$
\begin{aligned}
n^{2}\left(\mathrm{SiO}_{2}\right)= & +\frac{0.6961663 \lambda^{2}}{\lambda^{2}-(0.0684043)^{2}} \\
& +\frac{0.4079426 \lambda^{2}}{\lambda^{2}-(0.1162414)^{2}}+\frac{0.8974794 \lambda^{2}}{\lambda^{2}-(9.896161)^{2}}
\end{aligned}
$$

These refractive indices are plotted as functions of wavelength $\lambda$ in Fig. 3 in the $1.2-4-\mu \mathrm{m}$ spectral range. Here we are interested in the relative refractive index

$$
n=n(\mathrm{Si}) / n\left(\mathrm{SiO}_{2}\right)
$$

of the $\mathrm{Si}-\mathrm{SiO}_{2}$ interface. This relative index is plotted versus $\lambda$ in Fig. 4, which shows that $n=\sqrt{2}+1$ is satisfied at the two wavelengths of

$$
\lambda_{1}=1.4224 \mu \mathrm{m}, \quad \lambda_{2}=2.8644 \mu \mathrm{m} .
$$

The maximum deviation of $n$ from $\sqrt{2}+1=2.4142$ between these two wavelengths, $\Delta n_{\max }=-0.0132$, occurs at $\lambda=2 \mu \mathrm{m}$. Therefore $\mathrm{TIR}$ at the $\mathrm{Si}-\mathrm{SiO}_{2}$ interface at

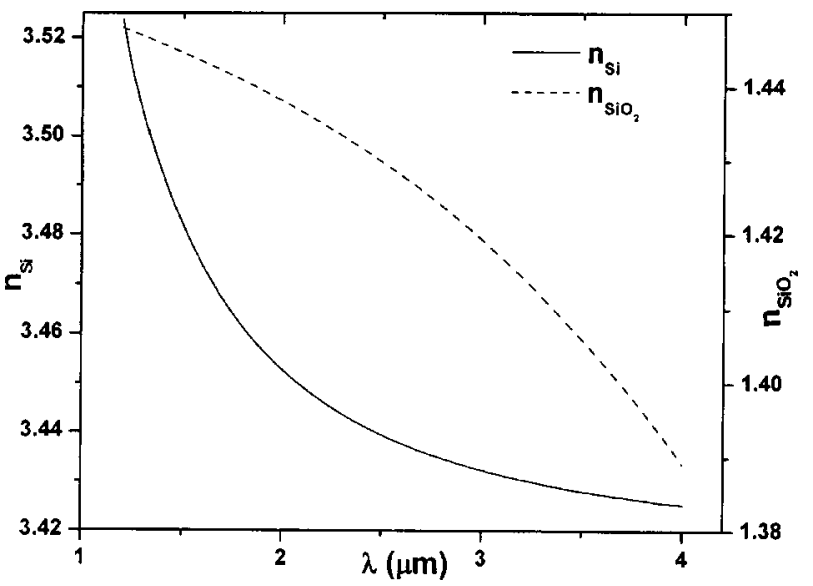

Fig. 3. Refractive indices of $\mathrm{Si}$ and $\mathrm{SiO}_{2}$ [given by Eqs. (5) and (6)] are plotted as functions of wavelength $\lambda$.

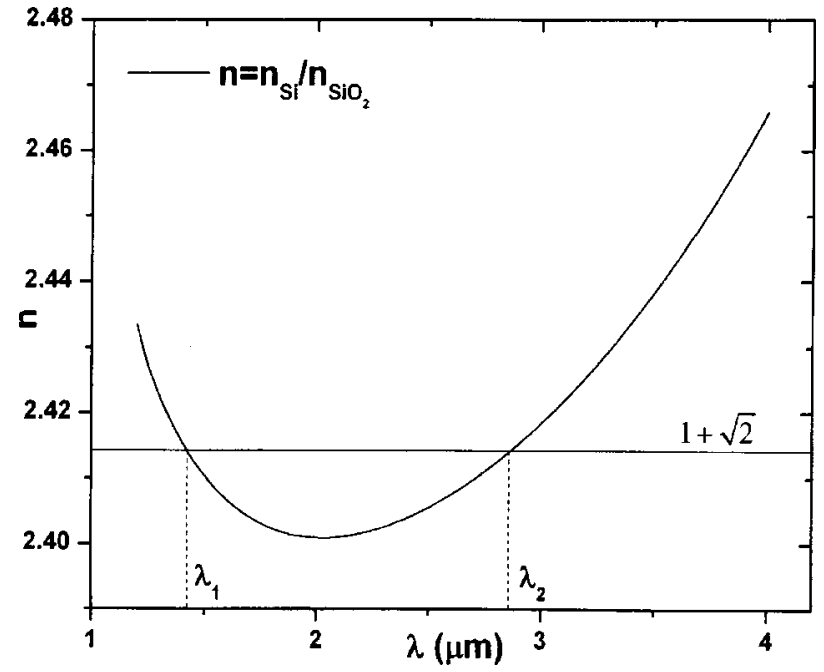

Fig. 4. Relative refractive index $n=n(\mathrm{Si}) / n\left(\mathrm{SiO}_{2}\right)$ [calculated by using Eqs. (5) and (6)] is plotted as a function of wavelength $\lambda$. Note that $n=\sqrt{2}+1$ at $\lambda_{1}=1.4224 \mu \mathrm{m}, \lambda_{2}=2.8644 \mu \mathrm{m}$. 


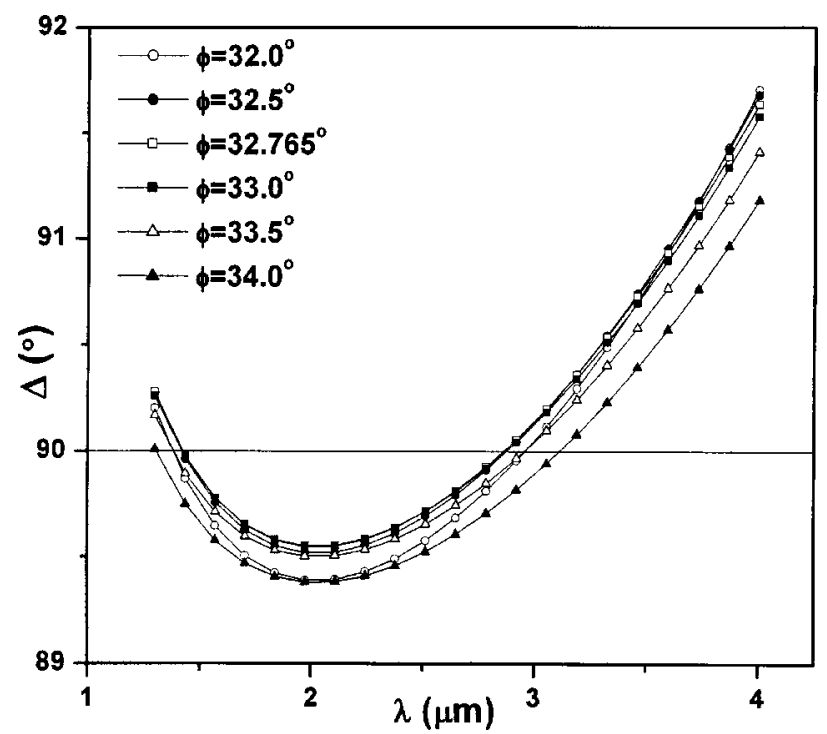

Fig. 5. Retardance $\Delta$ versus $\lambda$ for TIR at the $\mathrm{Si}-\mathrm{SiO}_{2}$ interface at five angles in the neighborhood of $\phi=32.765^{\circ}$.

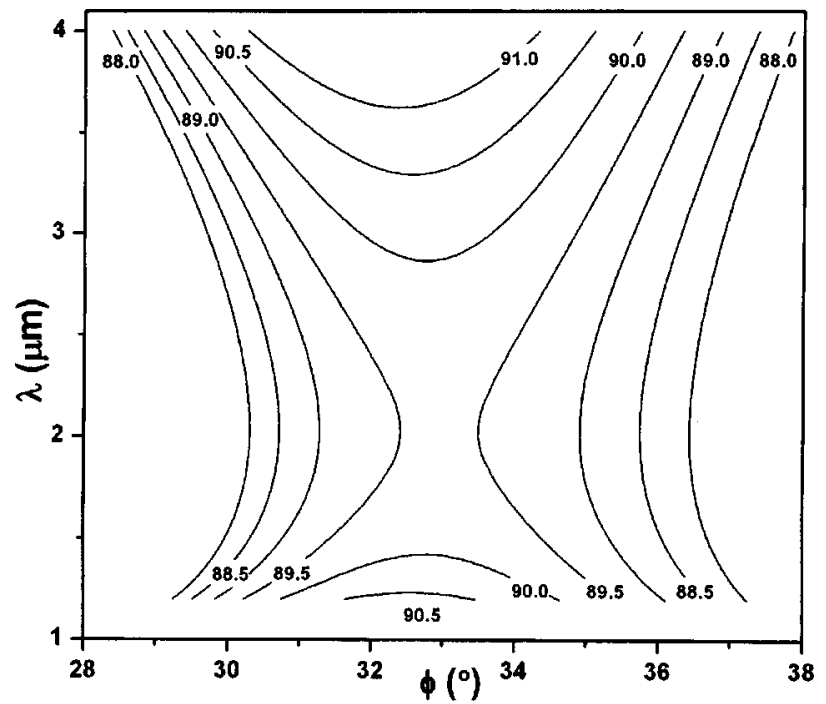

Fig. 6. Contours of constant $\Delta$ in the $\lambda-\phi$ parameter space, where $\Delta$ takes values between $88^{\circ}$ and $91^{\circ}$ in steps of $0.5^{\circ}$. This nomogram is constructed based on Eqs. (1) and (5)-(7).

and near $\phi=32.765^{\circ}$ provides achromatic quarter-wave retardation over a significant spectral range in the near and midinfrared.

\section{ANGULAR AND SPECTRAL RESPONSE OF THE $\mathrm{Si}-\mathrm{SiO}_{2}$ QUARTER-WAVE RETARDER}

Figure 5 shows $\Delta$ versus $\lambda$ at five angles of incidence in the neighborhood of $\phi=32.765^{\circ}$. Figure 5 indicates that the two wavelengths at which exact quarter-wave retardation is achieved are shifted as $\phi$ is changed.

Figure 6 is a map of constant- $\Delta$ contours in the $\lambda-\phi$ parameter space, where $\Delta$ takes values between $88^{\circ}$ and $91^{\circ}$ in steps of $0.5^{\circ}$. This design nomogram is constructed based on Eqs. (1) and (5)-(7). Figures 5 and 6 clearly demonstrate that our QWR is both reasonably achromatic and angle insensitive.

The angular insensitivity of the QWR that uses TIR at the $\mathrm{Si}-\mathrm{SiO}_{2}$ interface is even better than that implied by Figs. 5 and 6 . Because of the high refractive index of $\mathrm{Si}$, small variations of the internal angle of incidence, e.g., $\pm 3^{\circ}$ around $\phi=33^{\circ}$ as in Fig. 6 , are magnified by a factor equal to $n(\mathrm{Si}) \sim 3.5$ when one considers the refraction of light between air and Si. Therefore the field of view in air (outside the prism) is of the order of $\pm 10.5^{\circ}$ according to Snell's law. ${ }^{19}$

The thickness of the oxide layer at the base of the prism in Fig. 1 should be several times the penetration depth of light into the oxide at the longest wavelength in the spectral band of interest. (For example, a thickness $\geqslant 40 \mu \mathrm{m}$ is more than adequate in the $1-4-\mu \mathrm{m}$ spectral range.) This guarantees that the tail of the evanescent wave inside the film ${ }^{22}$ does not reach the outer surface of the oxide layer. This is essential to satisfy our assumption of a single buried interface and is also required for achieving device insensitivity to environmental contamination. Furthermore, to obtain the lowest possible insertion loss, the entrance and exit faces of the prism in Fig. 1 should be antireflection coated. $\mathrm{A} \mathrm{SiO}_{2}-\mathrm{Si}_{3} \mathrm{~N}_{4}$ bilayer can serve this function reasonably well. ${ }^{23}$

\section{CONCLUSION}

In summary, we have described a device that uses total internal reflection at a buried $\mathrm{Si}-\mathrm{SiO}_{2}$ interface at angles near $33^{\circ}$ to provide achromatic quarter-wave retardation in the near and midinfrared. The field of view in air is $\pm 10.5^{\circ}$ and the QWR is insensitive to environmental contamination. This device can be used in infrared ellipsometry ${ }^{24}$ and as a linear-to-circular or circular-tolinear polarization transformer in the $1.2-4-\mu \mathrm{m}$ spectral range where birefringent crystalline retarders are expensive or not widely available. This spectral range includes the important lightwave communication wave band and numerous tunable and discrete laser wavelengths, e.g., those of the HF, color-center, Er:glass, Er:YAG, Nd:YAG, Tm:YAG, and He-Ne lasers.

Finally, it will be of interest to search for other dielectric-dielectric interfaces (i.e., optical materials) that would perform a similar function in other spectral ranges.

R. M. A. Azzam can be reached by e-mail at razzam@uno.edu.

\section{REFERENCES}

1. W. A. Shurcliff, Polarized Light (Harvard University Press, Cambridge, Mass., 1962).

2. D. Clarke and J. F. Grainger, Polarized Light and Optical Measurement (Pergamon, New York, 1971).

3. R. M. A. Azzam and N. M. Bashara, Ellipsometry and Polarized Light (North-Holland, Amsterdam, 1987).

4. B. Boulbry, B. Bousquet, B. Le Jeune, Y. Guern, and J. Lotrian, "Polarization errors associated with zero-order achromatic quarter-wave plates in the whole visible spectral range," Opt. Express 9, 225-235 (2001). 
5. D. R. Solli, C. F. McCormick, R. Y. Chia, and J. M. Hickmann, "Birefringence in two-dimensional bulk photonic crystals applied to the construction of quarter-wave plates," Opt. Express 11, 125-133 (2003).

6. R. M. A. Azzam and F. A. Mahmoud, "Tilted bilayer membranes as simple transmission quarter-wave retardation plates," J. Opt. Soc. Am. A 18, 421-425 (2001)

7. R. M. A. Azzam and F. A. Mahmoud, "Symmetrically coated pellicle beam splitters for dual quarter-wave retardation in reflection and transmission,"Appl. Opt. 41, 235-238 (2002).

8. R. M. A. Azzam and A. De, "Circular polarization beam splitter that uses frustrated total internal reflection by embedded, symmetric, achiral multilayer coating," Opt. Lett. 28, 355-357 (2003)

9. R. C. Enger and S. K. Case, "Optical elements with ultrahigh spatial-frequency surface corrugation," Appl. Opt. 22 , 3220-3228 (1983).

10. A. G. Lopez and H. G. Craighead, "Wave-plate polarizing beam splitter based on a form-birefringent multilayer grating," Opt. Lett. 23, 1627-1629 (1998).

11. W. H. Southwell, "Multilayer coating design achieving a broadband $90^{\circ}$ phase shift," Appl. Opt. 19, 2688-2692 (1980).

12. J. H. Apfel, "Phase retardance of periodic multilayer mirrors," Appl. Opt. 21, 733-738 (1982).

13. E. Spiller, "Totally reflecting thin-film phase retarders," Appl. Opt. 23, 3544-3549 (1984).

14. M. M. K. Howlader and R. M. A. Azzam, "Periodic and quasiperiodic nonquarterwave multilayer coatings for 90-deg reflection phase retardance at 45-deg angle of incidence," Opt. Eng. 34, 869-875 (1995).

15. J. M. Bennett, "Critical evaluation of rhomb-type quarterwave retarders," Appl. Opt. 9, 2123-2129 (1970)

16. I. Filinski and T. Skettrup, "Achromatic phase retarders constructed from right-angle prisms: design,” Appl. Opt. 23, 2747-2751 (1984).

17. Technical Sessions "Optical Silicon I \& II," Conference program, 2002 OSA Annual Meeting, September 29-October 3, 2002, Orlando, Fla.

18. R. M. A. Azzam and M. M. K. Howlader, "Silicon-based polarization optics for the 1.30- and 1.55- $\mu \mathrm{m}$ communication wavelengths," J. Lightwave Technol. 14, 873-878 (1996).

19. M. Born and E. Wolf, Principles of Optics (Pergamon, New York, 1975).

20. R. M. A. Azzam, "Phase shifts that accompany total internal reflection at a dielectric-dielectric interface," J. Opt. Soc. Am. A 21, 1559-1563 (2004).

21. W. J. Tropf, M. E. Thomas, and T. J. Harris, "Properties of crystals and glasses," in Handbook of Optics, Vol. II, M. Bass, E. W. Van Stryland, D. R. Williams, and W. L. Wolfe, eds. (McGraw-Hill, New York, 1995), Chap. 33.

22. S. Zhu, A. W. Yu, D. Hawley, and R. Roy, "Frustrated total internal reflection: a demonstration and review," Am. J. Phys. 54, 601-607 (1986).

23. R. M. A. Azzam and K. Javily, "Antireflecting and polarizing transparent bilayer coatings on absorbing substrates at oblique incidence," Appl. Opt. 24, 519-526 (1985).

24. A. Röseler, Infrared Spectroscopic Ellipsometry (AkademieVerlag, Berlin, 1990). 